\title{
Evidence for self-sputtering during pulsed laser deposition of Zn
}

\author{
J. G. Hidalgo, R. Serna, E. Haro-Poniatowski*, and C.N. Afonso \\ Instituto de Optica, CSIC, Serrano 121, 28006 Madrid, Spain
}

Fax +34 915645557

e-mail: fjghidalg@io.cfmac.csic.es

\section{Abstract}

Thin films of $\mathrm{Zn}$ have been prepared by pulsed laser deposition by using a KrF excimer laser $(248 \mathrm{~nm})$. The laser energy density (E.D.) on the target has been varied in the 1 to $5 \mathrm{~J} \mathrm{~cm}^{-2}$ range. The results show that as the E.D. increases the material distribution changes. For low E.D. $\left(\leq 1.6 \mathrm{~J} \mathrm{~cm}^{-2}\right)$ the maximum of the distribution is at the substrate center, for intermediate E.D. it is displaced to the side, and a clear minimum appears at the center of the substrate for the higher E.D. $\left(\geq 4.5 \mathrm{~J} \mathrm{~cm}^{-2}\right)$. The growth velocity at the center of the substrate reaches a maximum value for E.D. of $2.8 \mathrm{~J} \mathrm{~cm}^{-2}$, and decreases for higher E.D. as a result of the competition between deposition and self-sputtering. Virtually a zero growth velocity is obtained for E.D. above $4.5 \mathrm{~J} \mathrm{~cm}^{-2}$. The selfsputtering process is most likely responsible for the increase of the film surface roughness as a function of the laser E.D. The low cohesive energy for metal $\mathrm{Zn}$, compared to other metals $(\mathrm{Fe}, \mathrm{Ag}, \mathrm{Cu})$ is correlated with the high efficiency of the selfsputtering for this material.

PACS: 81.15.Fg, 68.55.Ac, 68.37.-d

(*)Permanent address: Departamento de Física, CBI, Universidad Autónoma Metropolitana Iztapalapa, Apdo. Postal 55-534, México D.F., México 


\section{Introduction}

Metallic zinc films are interesting as coatings for protection of steel corrosion, as electrodes in solid dry-cell batteries, and more recently their use has been proposed for the development of nanometer scale photonic devices [1]. Pulsed laser deposition (PLD) is an attractive alternative preparation method. During (PLD) species with high kinetic energy that can exceed $100 \mathrm{eV}$ are ejected from the target. The mean kinetic energy of the species arriving at the substrate can thus be higher than the energy threshold for sputtering of most metals which is in the range $10-25 \mathrm{eV}$. Therefore self-sputtering (also called resputtering) from the growing film can occur [2]. Nevertheless there are few direct observations of sputtering effects [2, 3, 4, 5], except for the reports of Krebs and co-workers on sputtering of metals, mainly Fe and $\mathrm{Ag}[2,6,7,8,9]$. In those reports they show the importance of sputtering on the understanding of the growth kinetics.

The aim of this work is to study the role of self-sputtering during deposition of $\mathrm{Zn}$. To analyze the growth kinetics of $\mathrm{Zn}$, PLD has been performed at different energy densities, and the material distribution and growth velocity of the films have been studied as a function of the laser energy density. It will be shown how with a laser energy density of a few $\mathrm{J}$ per $\mathrm{cm}^{2}$, the self-sputtering of the $\mathrm{Zn}$ species arriving at the substrate is able to significantly modify the distribution of the material in the film and even prevent the film growth. Although sputtering is a general phenomenon addressed in PLD processes, this work shows that the self-sputtering process is specially efficient for the case of $\mathrm{Zn}$ due to its unique thermal properties and low cohesive energy. Therefore it is found that $\mathrm{Zn}$ can be considered as an excellent model case for the study of this phenomenon. 


\section{Experimental}

Thin films of $\mathrm{Zn}$ have been produced by pulsed laser deposition using an excimer laser $(\mathrm{KrF}, \lambda=248 \mathrm{~nm}, \tau=12 \mathrm{~ns}$ FWHM, $10 \mathrm{~Hz}$ repetition rate). The energy density (E.D.) at the target position has been varied from $1 \mathrm{~J} \mathrm{~cm}^{-2}$ to $5 \mathrm{~J} \mathrm{~cm}^{-2}$. The film growth was carried out in vacuum $\left(4 \times 10^{-5}\right.$ mbar). The substrate holder was located at 29 $\mathrm{mm}$ in front of the target and kept at room temperature. Three different substrates were placed simultaneously in front of the target: chemically-cleaned glass, Si wafer with native oxide coating and carbon coated mica. The glass substrate was located in the half upper part of the sample holder, and the $\mathrm{Si}$ and the mica substrates in the half lower part. Each type of substrate suits different characterization techniques for the same deposited film.

The reflectivity of the film during growth was monitored by using a HeNe laser (632.8 $\mathrm{nm}$ ) incident at the center of the substrate at $45^{\circ}$ on the Si wafer piece. The deposition was stopped when either the reflectivity reached a maximum or when it was reached a saturation value, whichever occurred first. The deposition times ranged from 200 to 700 s. To calibrate the film thickness two films deposited on $\mathrm{Si}$ were measured by Rutherford Backscattering Spectrometry (RBS). Scanning electron microscopy (SEM) was used to analyze the film surface morphology. The samples deposited on carbon coated-mica were floated off the substrate in deionised water and collected on microscope grids for transmission electron microscopy (TEM) observation.

The films deposited on glass were used to determine the two dimensional thickness distribution of the films from optical transmission measurements at $632.8 \mathrm{~nm}$ over an area of $22 \times 12 \mathrm{~mm}^{2}$. The thickness is calculated as $\mathbf{Z}=-\boldsymbol{\alpha}^{-1} \ln (\mathbf{T}), \boldsymbol{\alpha}$ is the absorption 
coefficient of $\mathrm{Zn}$ at $632.8 \mathrm{~nm}\left(\sim 810^{5} \mathrm{~cm}^{-1}\right)$, and $\mathbf{T}$ is the measured transmission. The thickness values obtained using this procedure are quite accurate for absorbing materials, as long as the thickness is smaller than the penetration depth of the light. The transmission of the films was measured immediately after deposition in order to prevent oxidation effects.

\section{Results}

Figure 1 shows the two dimensional thickness distribution of some selected $\mathrm{Zn}$ films where the lines represent points of equal thickness (values in $\mathrm{nm}$ are included in the figure). A gray color code has been added to the image, the darker areas corresponding to the thicker parts of the film. Only the upper half part of the total deposited area, corresponding to the glass substrate, is shown. For the lowest E.D., the maximum of the material distribution is approximately at the geometrical center of the substrate which is at the bottom center of the image. This is the expected distribution since the standard PLD geometry has been used, i.e. with the substrate in front of the target [10]. Nevertheless, as the laser E.D. is increased, it is observed that the maximum of the distribution shifts from the center to the right hand side of the image. For E.D. $4.5 \geq \mathrm{J}$ $\mathrm{cm}^{-2}$ a minimum is observed at the geometrical center of the substrate. Note that for the latter E.D., the maximum thickness reached at the center is only $7.2 \mathrm{~nm}$. From the evolution of the reflectivity measured as a function of the deposition time at the substrate center (not shown) it is observed that this thickness value was reached after 1000 pulses. Although the PLD process continued for another 1500 pulses the film thickness did not increase further, indicating that there was no effective film growth. The film deposited at $5.3 \mathrm{~J} \mathrm{~cm}^{2}$ showed the same behavior, but the final absolute value 
of the reflectivity was lower that is consistent with a thickness at the center of only 3 nm.

Figure 2 shows the growth velocity at the center of the substrate as a function of the laser E.D. for all the studied films. At E.D. $0.9 \mathrm{~J} \mathrm{~cm}^{-2}$, no significant deposition of material has been observed after 7150 pulses. For E.D. $1.3 \mathrm{~J} \mathrm{~cm}^{-2}$, the growth velocity is already close to $3 \times 10^{-3} \mathrm{~nm}$ per pulse. Thus the threshold E.D. for $\mathrm{Zn}$ deposition is in the range $0.9-1.3 \mathrm{~J} \mathrm{~cm}^{-2}$. As the E.D. increases up to $2.8 \mathrm{~J} \mathrm{~cm}^{-2}$ the growth velocity increases up to about $5.5 \times 10^{-3} \mathrm{~nm}$ per pulse. This growth velocity value is of the order of magnitude of that reported in our earlier works on $\mathrm{Cu}$ and $\mathrm{Ag}$ deposition for E.D. $2 \mathrm{~J}$ $\mathrm{cm}^{-2}[11,12,13]$. Above $2.8 \mathrm{~J} \mathrm{~cm}^{-2}$, the growth velocity steadily decreases and finally reaches mean effective growth velocity values that are virtually zero for E. D. $\geq 4.5 \mathrm{~J}$ $\mathrm{cm}^{-2}$. The decrease of the growth velocity for E.D. above $2.8 \mathrm{~J} \mathrm{~cm}^{-2}$ is in agreement with the fact that in Fig. 1(c), the thickness of the film shows a minimum at the center of the substrate. This result is in contrast with the growth velocities reported for other metals such as $\mathrm{Cu}, \mathrm{Ag}$ and $\mathrm{Fe}$ for E.D. in the range $4-10 \mathrm{~J} \mathrm{~cm}^{-2}$ which are usually quite large, typically $10^{-2} \mathrm{~nm}$ per pulse $[2,7,8]$.

Figure 3 shows the SEM images of some selected films taken from the film at the center of the substrate. The images show that there are nanometric size particles at the film surface. Statistical analysis of the mean diameter and distribution of the particles has been performed and the corresponding histograms are also shown in Fig. 3. The results show that the number of particles per unit area and the mean diameter of the particles increase as the E.D. is increased. The smoothest film, i.e. the one having less particles with smaller size, is obtained for the lowest E.D.. The roughness increases rapidly when 
the laser E.D. is increased, from 1.3 to $1.9 \mathrm{~J} \mathrm{~cm}^{-2}$. The TEM images (not shown) show that the films are polycrystalline and that the grain size (mean diameter) is in the range 5-30 nm. This size is of the same order of magnitude than the size of the particles observed by SEM. Therefore the particles observed by SEM are most likely Zn grains resulting from columnar growth (3D).

\section{Discussion}

To understand these results it should be realized that according to the used geometry the plasma plume is more intense, i.e. has a larger density of species and the species have a higher kinetic energy, at the position corresponding to the geometrical center of the substrate. The results thus evidence a competition between $\mathrm{Zn}$ deposition and selfsputtering of the film by the Zn high kinetic energy species arriving at the surface at this position. The deposition process is dominant for E.D. up to $2.8 \mathrm{~J} \mathrm{~cm}^{-2}$, whereas the selfsputtering dominates at higher energy densities, leading first to an effective decrease of the deposition rate, and finally to a $100 \%$ percent self-sputtering efficiency, i.e. no net deposition. This leads to the minimum in the material distribution observed in (Fig.1) at the geometrical center of the substrate. The roughness observed in the films can be correlated to this self-sputtering process. According to the SEM images in Fig. 3 smooth films with a low density of particles are only obtained for very low E.D. $\left(\leq 1.3 \mathrm{~J} \mathrm{~cm}^{-2}\right)$, for which very little self-sputtering takes place. When the E.D. increases, the selfsputtering becomes more important, ocurring most likely preferentially at the grain boundaries and thus enhancing the grains and making then more prominent in the SEM image. 
In order to understand why the self-sputtering is clearly evidenced for $\mathrm{Zn}$ at the typical E.D. used for ablation of metals, we have compared the properties of $\mathrm{Zn}$ to those of other metals for which the effect is not so strong when deposition is performed in similar conditions $[2,4,7,8]$. First it is worth pointing out that $\mathrm{Zn}$ has a low melting temperature that favors the generation of high kinetic energy species by laser ablation with low E.D. Since sputtering yield is known to increase with the kinetic energy of the incident species, it is expected that self-sputter effects in $\mathrm{Zn}$ should be noticeable for lower E.D.. Second, the cohesive energy of the metal is a measure of the strength of the energy binding of the atoms in the film, and it is found that $\mathrm{Zn}$ has a low cohesive energy, thus in principle it will be easier to sputter out deposited $\mathrm{Zn}$ species. The self sputter yields during PLD have been reported to be 0.55 for $\mathrm{Ag}$ and 0.17 for Fe [7] for energy densities in the range 4 to $10 \mathrm{~J} \mathrm{~cm}^{-2}$, these values being averaged over all atoms and ions within the plume and include reflection from the impinging species. Other authors have reported a sputtering yield of about 0.5 for $\mathrm{Cu}$ [4]. Fig. 4 shows a plot of the reported sputter yield for these metals at similar E.D. [2, 4, 7 and this work] as a function of the the cohesive energy of the metal [14]. The experimental data follows a linear trend that gives support to the fact that the low cohesive energy of the $\mathrm{Zn}$ compared to the other metals (up to a factor 3 compared to $\mathrm{Fe}$ ), is responsible for the high self-sputtering yield occurring in $\mathrm{Zn}$ for E.D. above $4.5 \mathrm{~J} \mathrm{~cm}^{-2}$ that completely inhibits Zn deposition.

\section{Conclusions}

It has been shown that self- sputtering is an important issue when preparing films by PLD since this process is able to completely prevent $\mathrm{Zn}$ film growth for E.D. above 4.5 $\mathrm{J} \mathrm{cm}^{-2}$. The self-sputtering process modifies the material distribution on the substrate, 
leading to unexpected inhomogeneous distributions. Finally the increase of the selfsputtering efficiency at high E.D. is responsible for the increase of the film surface roughness.

\section{Acknowledgements}

This work was partially supported by MCYT (Spain) under TIC2002-03235 project and by Consejo Nacional de Ciencia y Tecnologia de México (CONACYT). J. G. H. acknowledges a scholarship from the I3P program from the CSIC. E.H-P. thanks the Ministerio de Educación Cultura y Deporte of Spain for a sabbatical grant. I. Vickridge (UPMC, Paris, France) is acknowledged for RBS measurements. Española del Zinc S.A. (ZINSA) is gratefully acknowledged for providing the $\mathrm{Zn}$ target material through Manuel García. 


\section{Figure captions}

Figure 1. Two dimensional thickness distribution of the films deposited with E.D. of

(a) $1.3 \mathrm{~J} \mathrm{~cm}^{-2}$, (b) $3.9 \mathrm{~J} \mathrm{~cm}^{-2}$ and (c) $4.5 \mathrm{~J} \mathrm{~cm}^{-2}$ on glass substrates. Only the upper half part of the total deposited area is shown. The figure shows the lines of equal thickness (values included in the figure in $\mathrm{nm}$ ). Artificial color has been added in a gray scale. The darker color correspond to the thicker $\mathrm{Zn}$ areas.

Figure 2. Growth velocity of the films as a function of the laser E.D.. The line is a guide to the eye.

Figure 3. SEM images of the films deposited in vacuum with E.D. of (a) $1.3 \mathrm{~J} \mathrm{~cm}^{-2}$, (b) $1.9 \mathrm{~J} \mathrm{~cm}^{-2}$ and (c) $3.9 \mathrm{~J} \mathrm{~cm}^{-2}$ on the Si substrate. On the right of the images are shown the corresponding histograms of the number of particles as a function of its mean diameter.

Figure 4. Reported self-sputtering yields for $\mathrm{Ag}, \mathrm{Fe}, \mathrm{Cu}$ and $\mathrm{Zn}$ (this work) as a function of the cohesive energy. 


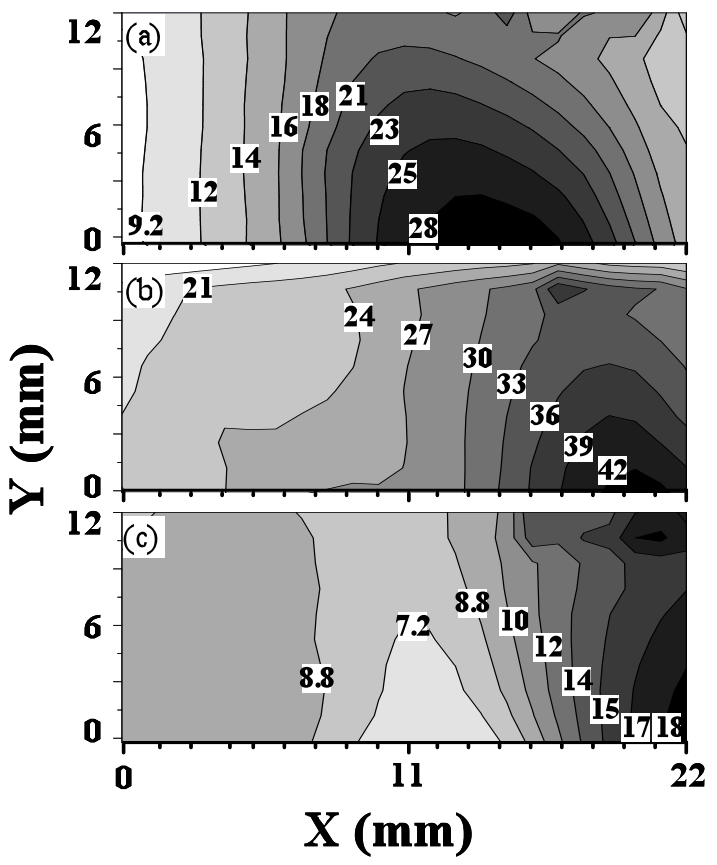

Figure 1 (of 4)

Evidence of self-sputtering..... by J.G. Hidalgo et al 


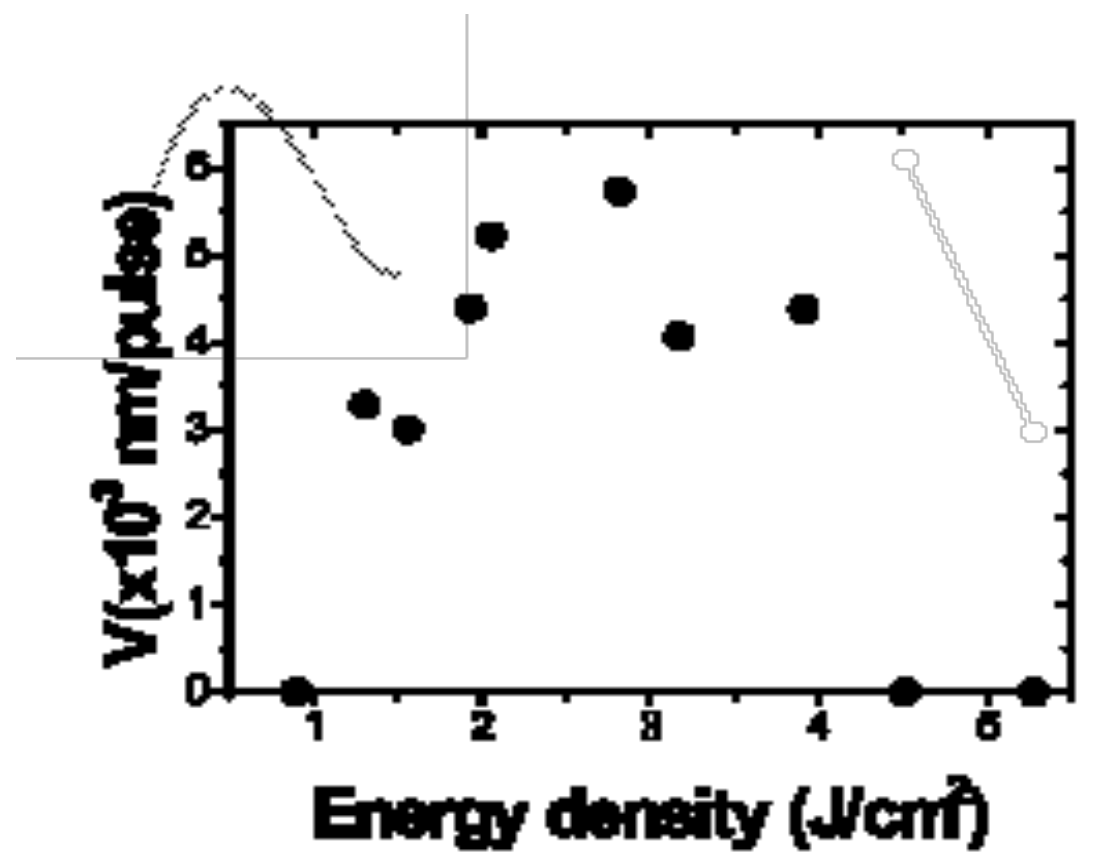

Figure 2 (of 4)

Evidence of self-sputtering..... by J.G. Hidalgo et al 

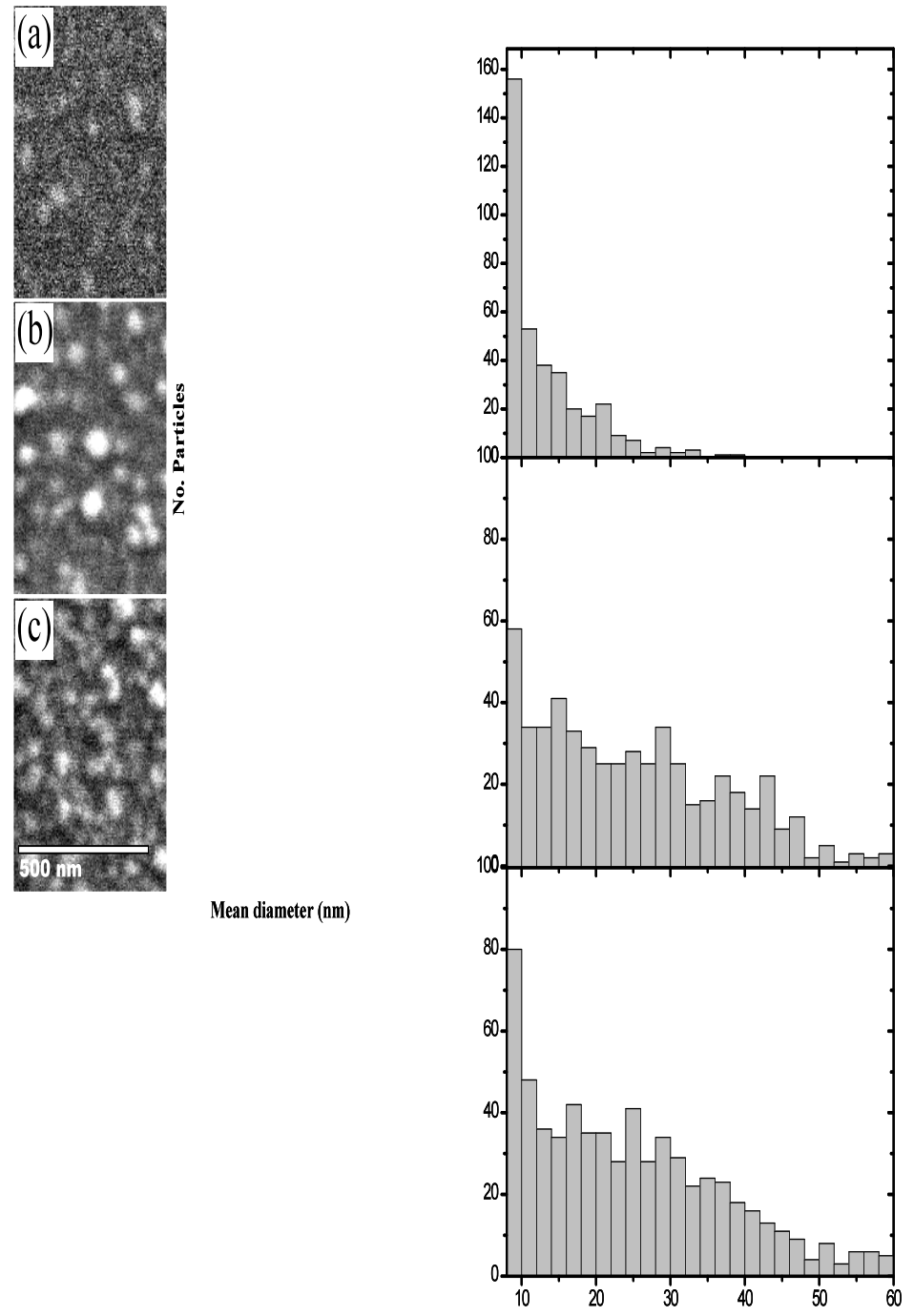

Figure 3 (of 4)

Evidence of self-sputtering..... by J.G. Hidalgo et al 


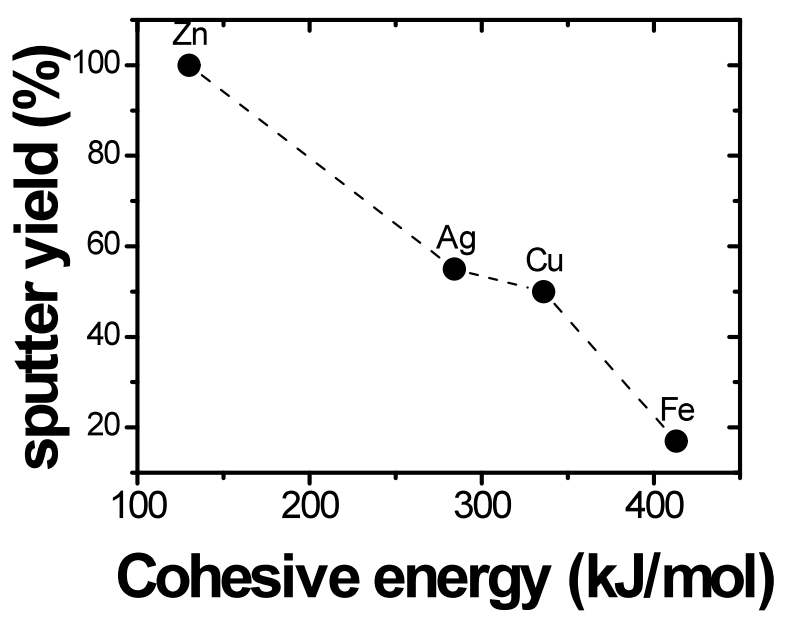

Figure 4 (of 4)

Evidence oof self-sputtering..... by J.G. Hidalgo et al 


\section{References}

1. T. Yatsui, S. Takubo, J. Lim, W. Nomura, M. Kourogi, and M. Ohtsu, Appl. Phys. Lett. 83, 1716 (2003)

2. S. Fahler, K. Sturm, H.-U. Krebs, Appl. Phys. Lett. 75, 3766 (1999), and references therin.

3. R. Jordan, D. Cole, J. Lunney, K. Mackay, and D. Givord, Appl. Surf. Sci. 86, 24 (1995)

4. J. Lunney, Appl. Surf. Sci. 86, 79 (1995).

5. S.K. Hau, K.H. Wong, P.W. Chan, C.L. Choy, Appl. Phys. Lett. 66, 245 (1995)

6. R. Gupta, M. Weisheit, H.-U. Krebs, P. Schaaf, Phys. Rev. B 67, 075402 (2003)

7. K. Sturm and H.-U. Krebs, J. Appl. Phys. 90, 1061 (2001).

8. H.-U. Krebs et al, Appl. Surf. Sci. 109/110, 563 (1997)

9. S. Fähler and H.-U. Krebs, Appl. Surf. Sci. 96-98, 61 (1996)

10. C.N. Afonso, R. Serna, F. Catalina, D. Bermejo, Appl. Surf. Sci. 46, 249 (1990).

11. R. Serna, C.N. Afonso, C. Ricolleau, Y. Wang, Y. Zheng, M. Gandais, I. Vickridge., Appl. Phys. A 71, 583-586 (2000)

12. C.N. Afonso, J. Gonzalo, R. Serna, J. C. G. de Sande, C. Ricolleau, C. Grigis, M. Gandais, D. E. Hole, and P. D. Townsend, Appl. Phys. A 69, S201-7 (1999)

13. J.P. Barnes, A. K. Petford-Long, R. C. Doole, R. Serna, J. Gonzalo, A. SuárezGarcía, C. N. Afonso, D. Hole, Nanotecnology 13, 465-470 (2002)

14. C. Kittel, Introduction to solid state physics, John Wiley \& sons, Inc., New York, Seventh Edition (1996), pg. 57. 